\title{
ARS en Políticas para la I nnovación Tecnológica: un enfoque teleológico
}

\author{
Silvia I. Almanza ${ }^{1}$ \\ Universidad Nacional Autónoma de México \\ Centro de Ciencias Aplicadas y Desarrollo Tecnológico \\ Rosa Luz González \\ Universidad Autónoma Metropolitana-Azcapotzalco \\ Hilda C. Hernández \\ Centro de Tecnología Avanzada CIATEQ - Querétaro
}

\section{Resumen}

Se presenta un novedoso enfoque de aplicación para el Análisis de Redes Sociales (ARS) dentro del campo de Estudios sobre Innovación, desde una perspectiva de Sistemas Complejos, para intervenir en el diseño e implementación de políticas de aprendizaje para la innovación, en países poco innovadores. Dos términos son introducidos, sistema de aprendizaje para la innovación y micro red de actividades intradiada, con el fin de integrar la noción teleológica dentro del ARS. Es ilustrado con un ejercicio de aplicación a nivel micro (organizacional) para un problema de planeación estratégica de la universidad pública: la falta de vinculación de sus organismos de investigación con el sector productivo. Se presenta un marco conceptual para el diseño de una herramienta de evaluación institucional que permita captar la aptitud de vinculación actual y la percepción de la visión en este tipo de organizaciones (fuerzas y debilidades). El objetivo es introducir buenas prácticas de vinculación y así, eventualmente favorecer la articulación de un sistema de transición de aprendizaje para la innovación. La congruencia en el diseño de la herramienta de evaluación (cuestionario) para vincular el quien con el cómo y el para qué, es ilustrada con una muestra de preguntas. Algunas implicaciones de esta propuesta son discutidas, tanto desde el punto de vista de la planeación estratégica como del ARS.

Palabras clave: ARS enfoque teleológico - sistemas complejos - herramienta de evaluación institucional - sistema de transición de aprendizaje para la innovación micro redes de actividades intradiada.

\begin{abstract}
This paper presents a novel approach of application for Social Nertwork Analysis (SNA) into the field of Innovation Studies from a Complex Systems view, with the aim to intervene in the design and implementation of learning for innovation policy, in less innovative countries. Two terms are introduced, learning system for innovation and micro-network of activities intra-dyad, in order to integrate the teleological notion into the SNA. It is illustrated with a micro-level exercise (institutional performance evaluation of a $R \& D$ Centre) for a public university strategic problem: the lack of links of their research organizations with the productive sector. A conceptual framework is presented for the design of an institutional evaluation tool to catch the linkage aptitude and vision in this type of organizations (strengths and weaknesses), with the aim to intervene for the change, in order to increase the linkage potentiality, and so, eventually increase the potentiality to articulate a Learning for Innovation Transition System. The congruence in the design of the evaluation tool (questionnaire) to tie in the who with the how and the what for (the strategic objectives of the organization), is illustrated
\end{abstract}

${ }^{1}$ Enviar correspondencia a: Silvia I. Almanza M., silviaa@servidor.unam.mx; silvia.almanza@ccadet.unam.mx; 
REDES- Revista hispana para el análisis de redes sociales

Vol.17,\#3, Diciembre 2009

http: // revista-redes. rediris.es

with a sample of questions. Some implications of this proposal are argued, either from the point of view of strategic planning as from the SNA.

Key words: ARS teleological approach - complex systems - institutional evaluation tool - Learning for Innovation Transition System - micro-network of activities intradyad.

\section{Introducción}

Aunque el flujo de conocimiento científico y tecnológico ha jugado un importante papel a lo largo de la historia de la humanidad, hoy más que nunca en el mundo se percibe la necesidad de basar el desarrollo y el crecimiento económico en el uso eficiente del conocimiento y la innovación. Para entender y potenciar la capacidad innovadora, un enfoque ampliamente aceptado entre los estudiosos del fenómeno de la innovación es el sistémico. El enfoque de sistemas de innovación, sin embargo, tuvo en su origen una connotación ex post, para estudiar aquellos sistemas razonablemente articulados en países avanzados, donde históricamente se ha desarrollado una cultura de innovación.

Por el contrario, en países tradicionalmente poco innovadores como México, no puede hablarse propiamente de sistemas de innovación (Arocena y Sutz, 2002): los actores están atomizados, el ambiente es cambiante, incierto y a menudo contradictorio; la cultura innovadora es escasa, las sinergias esperables y las propiedades emergentes que caracterizan a un sistema de innovación están ausentes. Con todo, es evidente que las presiones para ponerse al día y acoplarse con el acelerado avance tecnológico mundial (catching up) les presiona a querer cambiar ese estado en el que se encuentran, a buscar maneras efectivas de capturar y hacer un uso eficiente del conocimiento y a querer llegar a tener sistemas de innovación razonablemente articulados. Para ello, los actores, secularmente poco vinculados, han de aprender a interaccionar efectivamente y a construir aquellas redes requeridas para movilizar el conocimiento cada vez más complejo (David y Foray, 1995).

El concepto sistema de innovación, por lo tanto, no puede adoptarse mecánicamente en los distintos contextos. Ha de ser adaptado a una realidad en donde mucho está aun por construirse, muchas de esas interacciones han de ser inducidas y ciertos usos y costumbres, modificados. A diferencia de los países avanzados, en estos otros implica abordar el problema desde una perspectiva ex ante, para favorecer, en una primera fase, el proceso de articulación de un sistema de transición. A este sistema le llamaremos aquí sistema de aprendizaje para la innovación-, que permita pasar eventualmente a otro estado superior, el sistema de innovación propiamente. Esto es importante, porque aprender a innovar (learning before doing) es la gran 
REDES- Revista hispana para el análisis de redes sociales

Vol.17,\#3, Diciembre 2009

http: //revista-redes. rediris.es

necesidad en muchos de estos países que buscan el crecimiento económico y deseablemente, el desarrollo social a través del uso efectivo del conocimiento.

La conformación de sistemas de aprendizaje para la innovación lleva implícita la idea de fortalecer la capacidad de absorción tecnológica nacional, para incrementar el potencial de captura tecnológica. La respuesta de las instituciones de educación superior (IES) y sus centros de I+D (CIUs) a esa necesidad, de cara a la propuesta de desarrollo basado en conocimiento es un gran desafío, que requiere de una cuidadosa planificación en sus políticas de vinculación.

Frente a esto, un problema básico de política es tener claro cómo instrumentarla para inducir el cambio. En el caso de la desvinculación ciencia - industria, un problema de planeación desde un enfoque sistémico es aportar elementos que les permitan a los CIUs incrementar su nivel de aptitud para transferir y difundir conocimiento al sector productivo. Ello implica no perder de vista tanto su contribución a la base de conocimiento científico, como su papel en el incremento del potencial de la capacidad de absorción tecnológica nacional. Para un $\mathrm{CIU}$, el primer paso en este camino es obtener un diagnóstico de su situación actual y una visión de la deseable. Conocer las fortalezas y debilidades de cada organización, es un requerimiento obligado para implantar las medidas que conducirán al cambio con un sentido de dirección - en este caso, para potenciar su aptitud de vinculación. El diseño de metodologías e instrumentos adecuados para el diagnóstico y la intervención es tanto un requerimiento para los tomadores de decisiones como un reto para los estudiosos del campo de la política de innovación en países en desarrollo.

Surge de aquí la motivación que dio origen al proyecto en el que se inserta el presente trabajo, orientado a diseñar una herramienta de planeación estratégica que permita detectar las fuerzas y debilidades institucionales de los $\mathrm{ClUs}$ en relación con su aptitud para responder a las necesidades del país ${ }^{2}$. Los criterios para su diseño fueron: a) que permitiera hacer el mencionado diagnóstico; b) que sirviera como una herramienta de intervención para orientar el aprendizaje organizacional y una guía para sus prácticas y actividades - para favorecer la producción y transferencia efectiva de conocimiento -, con un sentido de previsión; c) que tomara en cuenta el potencial de contribución de este tipo de organizaciones dentro del proceso de fortalecimiento de la capacidad de absorción tecnológica nacional, dentro de un esquema global de transición, "sistema de aprendizaje para la innovación".

${ }^{2}$ EI Marco Conceptual desarrollado para todo el proyecto se encuentra en Almanza y Hernández, 2007. Este Instrumento ha de ser complementado con estudios sobre amenazas y oportunidades, para obtener un cuadro completo de la situación. 
REDES- Revista hispana para el análisis de redes sociales

Vol.17,\#3, Diciembre 2009

http: // revista-redes. rediris. es

Para el diseño del instrumento se contempló desde su inicio la posibilidad de integrar el herramental que ofrece el Análisis de Redes Sociales (ARS) con otras metodologías de planeación - como el Benchmarking-, por las potencialidades que ofrece en cuanto a modelación, representación visual y capacidad de integración de distintos niveles analíticos a través de las interconexiones entre los actores de un sistema ${ }^{3}$ (Almanza et al, 2004). El reto era, sin embargo, que en planeación importa conocer no sólo el "quién se relaciona con quién", sino el "cómo hacer que se relacione quién con quién y para qué". Esto planteaba el requerimiento metodológico de capturar y vislumbrar el aspecto dinámico del cambio desde su base, entender cómo se establecen o dejan de establecerse cada una de las interacciones de un CIU con otros actores (diadas), a través de ciertas prácticas y actividades, para intervenir en su corrección. Desde el punto de vista de la visión de un CIU y de su papel dentro del sistema, las prácticas y actividades han de orientarse hacia ciertos objetivos estratégicos para poder establecer el vínculo. Algunas de ellas han probado ser más exitosas que otras (las "buenas prácticas"), en tanto que otras son intrascendentes o pueden tener efectos negativos para un propósito dado. Para un $\mathrm{CIU}$, el establecimiento estratégico de vínculos es crucial para permitir el flujo de conocimiento: a medida que la producción de conocimiento científico y tecnológico se vuelve más compleja y la interdisciplina toma más importancia, los canales más efectivos para el intercambio son las redes de conocimiento.

La pregunta era si el herramental del ARS podría aplicarse bajo el enfoque teleológico ${ }^{4}$ requerido en planeación desde la perspectiva de sistemas complejos. Como se ha dicho, el ARS presta más atención al estudio de las estructuras sociales, insistiendo menos en el cómo, en el porqué y para qué la gente hace lo que hace, y más en la comprensión de las condicionantes estructurales de sus acciones (Sanz Menéndez, 2003). Ello nos condujo a buscar un nivel de análisis más fino, a nivel de diada, y a proponer el concepto de micro redes de actividades intradiada.

El concepto micro redes de actividades intradiada nos permite traducir al lenguaje del ARS los objetivos estratégicos, prácticas y actividades que pueden generar las diadas, así como dar consistencia al diseño del instrumento de evaluación institucional dentro de este enfoque. El objetivo de este trabajo es presentar el concepto arriba mencionado y su expresión operativa en el diseño de un instrumento para la intervención hacia el cambio. Al intervenir en la diada se va integrando la red egocentrada de un CIU, que con el tiempo llegará a conformar la red total

\footnotetext{
${ }^{3}$ EI ARS generalmente estudia la conducta de los actores a nivel micro, los patrones de relaciones, la estructura de la red a nivel macro y las interacciones entre los dos niveles (Sanz Menéndez, 2003).

${ }^{4}$ Es decir orientado a objetivos, según el enfoque de Dinámica de Sistemas Complejos.
} 
REDES- Revista hispana para el análisis de redes sociales

Vol.17,\#3, Diciembre 2009

http: // revista-redes. rediris.es

(Figura 1). Tanto en el proceso de formación de dicha red como una vez que ésta se ha constituido, el ARS permite además la aplicación de herramientas que facilitan el análisis y la intervención.

\section{El ARS como enfoque}

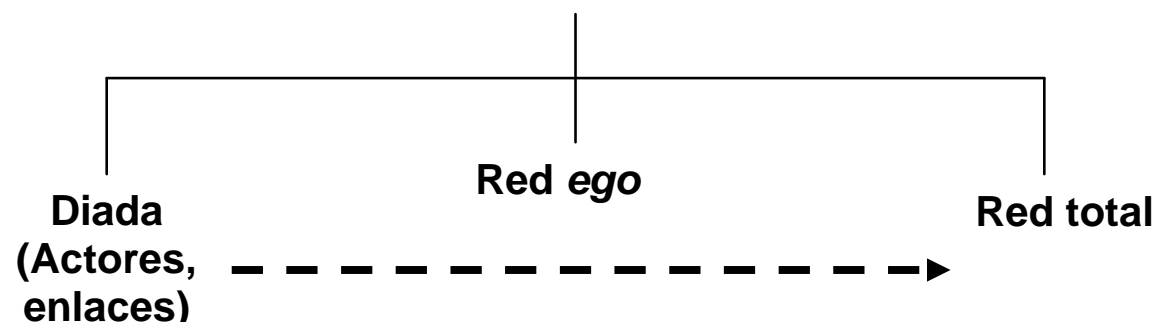

Figura 1. Proceso de integración de la red.

El diseño del instrumento se sustenta en el enfoque de Sistemas Complejos. Se le ha integrado el herramental del ARS para modelación, visualización, conexión de niveles de análisis e identificación de estructuras relacionales a las que se puedan atribuir propiedades sistémicas, que pueden ayudar a comprender, predecir y gestionar mejor los resultados de la acción de los actores involucrados (Conway et al, 2001; Sanz Menéndez, 2003).

El trabajo comprende cuatro partes. En la primera se presenta el problema de la desvinculación ciencia - industria en países poco innovadores, como México. En la segunda, se hace un intento de integración conceptual de sistemas complejos de innovación y ARS; se despliega el concepto sistemas de aprendizaje para la innovación - el cual se centra en un nivel de análisis micro más fino -, para profundizar en lo que ocurre al interior de la diada y abordar desde ahí el problema de cómo incrementar el potencial de vinculación entre un actor ego (CIU) y sus alters. Para ello se introduce el concepto micro redes de actividades intradiada. En la tercera parte se presentan aspectos que sustentan el diseño de la herramienta, de la metodología y diseño del cuestionario para la recopilación de datos -tanto de atributos ${ }^{5}$ como de relaciones entre actores-, que permitan generar las estructuras básicas de información para representar una red social (gráficas y matrices). En la cuarta parte se habla de las implicaciones de esta integración, tanto para el ARS como para la planeación estratégica.

5 En interacciones complejas como las que se presentan en vinculación ciencia industria, los atributos de los actores pueden ser prerrequisito para o resultado de las relaciones que se generan entre los diferentes actores 
REDES- Revista hispana para el análisis de redes sociales

Vol.17,\#3, Diciembre 2009

http: // revista-redes. rediris.es

\section{El Problema de la Desvinculación}

Ante el aceleramiento del avance tecnológico mundial, uno de los problemas sustantivos que actualmente enfrentan los países poco innovadores como México y otros países latinoamericanos, es la pérdida abrupta de su posición competitiva, con serias implicaciones para el crecimiento económico y el desarrollo social.

La dinámica de competencia industrial contemporánea requiere de un proceso de desarrollo científico y tecnológico estratégico que se enfoque en la ventaja competitiva. Según el Foro Económico Mundial, México presenta un rezago constante en desarrollo tecnológico, muy por detrás de países como Brasil y los de la OCDE. En el índice de competitividad 2004, México superaba la posición 30 en la mitad de los factores de competitividad. En 2006 solamente supera el lugar 30 en el subíndice: Sectores económicos con potencial. Así, México pasa a ocupar el lugar 33 de 45 países, mostrando un retroceso de tres posiciones, para colocarse entre los seis países que más posiciones perdieron y retrocede posiciones en seis de los 10 factores de competitividad (Porter et al, 2007).

Una de las causas de este problema es la falta histórica de una política nacional de desarrollo basado en conocimiento, que en los hechos se expresa en la atomización de los actores potenciales, en la falta de priorización de áreas estratégicas de $I+D$, en una actividad investigadora orientada exclusivamente a la calidad científica y una cultura poco innovadora del sector productivo nacional. Todo ello se refleja en la limitada capacidad de captura tecnológica nacional, requerida para contender con el empuje del avance científico y tecnológico mundial (etapa de pre catching up, Narula, 2004). En este contexto, la limitada capacidad de absorción tecnológica del sector productivo nacional aparece como una de las barreras más importantes para superar esta etapa.

La capacidad de absorción es un proceso evolutivo, que define en gran medida, el nivel de aptitud de un sistema, para innovar. En ausencia de sistemas de innovación bien articulados, la capacidad de absorción tiende a declinar. Desde esta perspectiva, puede decirse que la atomización y ausencia de sistemas de innovación explica, en gran medida, la situación de atraso de los países poco innovadores y su consecuente pérdida de competitividad.

Una expresión a nivel micro de esta atomización se manifiesta en el desacoplamiento entre las cambiantes necesidades del sector productivo nacional y la producción, así como una visión estrecha de la transferencia de conocimiento de los centros universitarios de I+D. Ese es el problema de la desvinculación (Mansfield and Lee, 1996; Mansfield, 1997). Para modificar esta situación de alejamiento, una política de 
REDES- Revista hispana para el análisis de redes sociales

Vol.17,\#3, Diciembre 2009

http: // revista-redes. rediris.es

vinculación incluyente ha de contemplar dentro del objetivo general de transferir conocimiento relevante a la sociedad, ciertos objetivos estratégicos inmediatos para orientar la producción de conocimiento hacia la calidad social o pertinencia (van der Meulen y Rip, 2000). Entre ellos se pueden destacar el desarrollo de lazos perdurables, la generación de un clima de confianza y credibilidad, la comunicación adecuada para propiciar un lenguaje común, etc. Trabajar en estos objetivos sentará las bases para la articulación a futuro, de un verdadero sistema de innovación.

\section{I ntegración Conceptual Redes - Planeación}

Este trabajo aborda un problema de política universitaria en países poco innovadores: cómo inducir la vinculación entre un actor que produce o adapta conocimiento ( $\mathrm{CIU}$ - actor ego) y los potenciales grupos de interés (alters) con los que debiera interactuar para que fluya el conocimiento (receptores, beneficiarios, patrocinadores, clientes o proveedores). En un contexto de ausencia de sistemas de innovación articulados - típico de países poco innovadores-, se propone un enfoque de política sistémico, orientado al aprendizaje para la innovación, como paso de transición hacia el engranaje y articulación de sistemas de innovación efectivos. Aprender a innovar, en este caso, significa aprender a interaccionar y a construir redes. Desde la perspectiva de sistemas complejos, se argumenta aquí, significa la posibilidad de intervenir desde la base, a nivel micro, para detonar el cambio a nivel meso y macro.

\section{I nnovación y Redes}

El concepto innovación tecnológica se refiere a aquellas actividades creativas relacionadas con la introducción de nuevas ideas, prácticas u objetos a un mercado o comunidad (Sundbo, 1998). Para que ocurra el intercambio de conocimiento del que se nutre la innovación, se requiere de la interacción e interdependencia de muy diversos grupos de interés (actores) de manera sistémica (Dodgson, 2000). A medida que se incrementa la complejidad en la producción de conocimiento, el desarrollo tecnológico y los procesos de innovación, se multiplican las competencias requeridas. Es claro que para contender con sistemas tecnológicos cada vez más complejos - que requieren de una gran diversidad de bases de conocimiento -, las capacidades individuales aisladas no funcionan. Capacidades y competencias funcionalmente interrelacionadas y continuamente actualizadas para permitir el trabajo interdisciplinario, en equipo, son requeridas cada vez más para contender con el acelerado cambio tecnológico mundial. La creación y funcionamiento de redes para desarrollar e integrar capacidades relevantes se vislumbra así, como la mejor solución para enfrentar la creciente complejidad científica y tecnológica. De aquí la 
REDES- Revista hispana para el análisis de redes sociales

Vol.17,\#3, Diciembre 2009

http: // revista-redes. rediris.es

importancia del carácter sistémico de la innovación, de las redes y de su principal propiedad emergente, el aprendizaje (Rycroft y Kash, 1999).

Un sistema de innovación está integrado por individuos y organizaciones que directa o indirectamente invierten tiempo y energía en la producción de conocimiento técnico y científico para uso comercial. Los actores pertenecientes al sistema tienen al menos un papel que cumplir: el gobierno primordialmente como orquestador; el sector industrial como productor de bienes y servicios; la universidad como productora y transmisora de conocimiento, etc. De las misiones o intereses de los actores, surgen sus objetivos y motivaciones para interactuar. De acuerdo con ellas, establecen sus reglas, rutinas, prácticas y actividades a través de las cuales fluye y se recombina el conocimiento de forma compleja, a manera de redes (Kline and Rosenberg, 1986). La organización de la red da forma al proceso de innovación tecnológica, y esa innovación tecnológica da forma a los cambios subsecuentes en la red. Este proceso coevolutivo es en sí, un proceso de aprendizaje, y es considerado como la propiedad emergente más importante de los sistemas de innovación (Rycroft \& Kash, 1999; Lundvall, 1988; 1996; 1996a; Lundvall y Borras, 1999; Lundvall, 2004).

Como se señaló antes, en los países poco innovadores los actores suelen estar atomizados. Sus instituciones, normas, rutinas, prácticas y actividades a menudo no son las más adecuadas para favorecer la innovación: no existen las condiciones objetivas y subjetivas para innovar, ni aparecen las propiedades emergentes propias de los sistemas de innovación. Dar por hecho su existencia en estos países - con historias y contextos diferentes al de países con sistemas de innovación ya articulados -, como punto de partida para el análisis o el diseño de políticas, puede ser una falacia que conduzca a costosos errores y a obtener resultados no deseados. Con base en esta reflexión, en este trabajo se adopta una actitud más realista: se propone orientar las políticas hacia la articulación de sistemas de aprendizaje para la innovación, en donde el objetivo sería la construcción de la capacidad de absorción tecnológica del sector productivo nacional, desde un estado de transición hacia la articulación de sistemas de innovación.

\section{Capacidad de Absorción y Redes}

La capacidad de un CIU para responder a las necesidades de la sociedad, es la esencia de la vinculación. Desde el punto de vista sistémico, el papel de los CIUs es capturar, producir transferir y difundir conocimiento. Este flujo de conocimiento conlleva un proceso de aprendizaje interactivo, en donde productores y receptores van adquiriendo progresivamente, mayores capacidades y competencias para captar 
REDES- Revista hispana para el análisis de redes sociales

Vol.17,\#3, Diciembre 2009

http: // revista-redes. rediris.es

y utilizar el conocimiento cada vez más complejo, que esté potencialmente disponible.

El conocimiento potencialmente transferible desde los CIUs suele ir incorporado de maneras muy diversas: en publicaciones, en patentes, en recursos humanos calificados, en nueva instrumentación y metodologías, en solución a problemas complejos, etc. (Arnold, 1998). Así, las competencias y habilidades de un CIU para producir, incorporar, transferir y difundir el conocimiento relevante desde diversos frentes definirán en gran medida, su aptitud para vincularse. Desde el punto de vista de la producción de conocimiento relevante, la interacción y participación en redes con otros actores es fundamental para sostenerse dentro del estado del arte así como para el desarrollo de proyectos cada vez más complejos. Así, la transferencia del conocimiento ya producido e incorporado por ejemplo, en recursos humanos calificados, la movilidad de éstos hacia y desde el sector productivo es uno de los medios más efectivos para el incremento de la capacidad de absorción tecnológica nacional.

Por otro lado, las capacidades y competencias requeridas para enfrentar la complejidad científica y tecnológica requieren cada vez más de la interacción de actores con bases de conocimiento muy diversas. Ellas son las que permitirán adaptarse rápidamente a las oportunidades cambiantes (Prahalad y Hammel, 1990). En un esquema de aprendizaje para la innovación, la participación en redes para adquirir capacidades adquiere particular importancia. En una etapa de transición, el objetivo no es tanto la producción de innovaciones tecnológicas radicales como tales, sino la construcción de capacidades y habilidades. Este esquema de redes de adquisición de capacidades para la innovación podría contribuir a incrementar el potencial de la capacidad de absorción, al abordar progresivamente problemas complejos desde una visión interdisciplinaria. Por la lógica misma de las cosas, es de esperarse que la participación en este tipo de redes incremente las capacidades y habilidades, por el proceso mismo de aprendizaje, para evolucionar progresivamente a redes de innovación propiamente, a medida que se incremente la capacidad de absorción colectiva.

Por otro lado, de acuerdo con Rycroft y Kash (1999), las redes de innovación crecientemente adquieren patrones de interacción que son auto-referenciados. Estos sistemas socio técnicos no pueden entrar en relaciones que no sean en parte basadas en los patrones de acciones que las definen y que las han definido a lo largo de la historia. Esta consideración, desde el punto de vista de una estrategia de aprendizaje para la innovación, es de gran importancia: inducir el 
REDES- Revista hispana para el análisis de redes sociales

Vol.17,\#3, Diciembre 2009

http: // revista-redes. rediris.es

establecimiento del aprendizaje y la colaboración como patrón de acciones a través del fomento de redes (innovación abierta) es inducir una nueva cultura, más abierta a integrar distintos tipos de conocimiento y formas de abordar problemas, que prepararán el terreno para la articulación de sistemas de innovación.

Con base en estos razonamientos, se puede pensar que la aptitud de un centro de investigación para responder a las necesidades de la sociedad ( $y$ por lo tanto, para producir conocimiento relevante) puede medirse por el tipo y calidad de los vínculos que sea capaz de generar a su alrededor en el tiempo, a manera de "red ego".

\section{Vinculación como Proceso de Aprendizaje y Redes}

Para que fluya el conocimiento a / desde la sociedad, es necesario que responda a una necesidad. La capacidad de identificar y traducir necesidades en problemas de investigación y desarrollo (I+D), y la capacidad para transferir y absorber el nuevo conocimiento por los diferentes actores, es lo que hace crucial el tema de la vinculación ciencia - industria, entendida como proceso de aprendizaje interactivo. Para que éste ocurra, los vínculos han de ser perdurables y la I+D, de calidad social (o relevante). Estos objetivos - que llamamos estratégicos -, se sustentan en la confianza, la credibilidad y el establecimiento de un lenguaje común, y pueden construirse a través de la institucionalización de ciertas prácticas y actividades.

En los centros de investigación, la manera de producir y transferir conocimiento - es decir, de vincularse - a través de sus prácticas, determinará en gran medida no sólo su propia fortaleza institucional, sino deseablemente, favorecerá el engranaje y articulación del sistema mismo de innovación, a nivel meso y macro.

\section{EI Modelo de Sistema de Aprendizaje para la I nnovación}

La dinámica del sistema de innovación, como se sabe, está determinada en gran medida por el acoplamiento entre la oportunidad técnica y las necesidades del usuario a través de las interacciones y relaciones entre los diversos actores. El éxito de las empresas y los individuos depende en gran medida de sus habilidades para adquirir rápidamente competencias a medida que son confrontados con nuevos problemas (Lundvall, 1985; Lundvall y Borras, 1999; Lundvall, 2004). Por ello, un sistema de aprendizaje para la innovación bien articulado, favorecería el acoplamiento del sector productivo con el cambio tecnológico mundial (catching up; Narula, 2004). En este sentido, un CIU puede contribuir no sólo produciendo conocimiento científico de vanguardia, sino de calidad social; y generar el capital humano adecuado, para contribuir activamente a la construcción de la capacidad de absorción tecnológica del sector productivo nacional mediante la implantación de buenas prácticas (Daghfous, 2004; van del Meulen y Rip, 2000). Para ello, es 
REDES- Revista hispana para el análisis de redes sociales

Vol.17,\#3, Diciembre 2009

http: // revista-redes. rediris.es

importante generar lazos perdurables con sus grupos de interés, entender su lenguaje, etc. Objetivos éstos que, junto con otros, permitirán a todos estar en mejor posición para aprovechar efectivamente las oportunidades tecnológicas potencialmente disponibles en el mercado. El diseño de una política de vinculación en este sentido implica asumir, como punto de partida, el aprendizaje para la innovación, como proceso social de interacción clave, asociado con la formación de redes, considerando la historia y el contexto particulares.

El enfoque de Sistema de Aprendizaje para la Innovación que aquí se propone, pone el énfasis en la situación de transición, a través de un objetivo (es decir, aprender a interactuar) en donde el horizonte vislumbrado es un Sistema de Innovación eficiente. Cada actor - desde sus distintas funciones-, hace su aportación para favorecer una dinámica de aprendizaje. A medida que se vayan articulando los distintos tipos de conocimiento entre los actores, el potencial de la capacidad de absorción tecnológica se irá incrementando progresivamente, dentro de un proceso coevolutivo. Esto se traducirá globalmente, en un sistema más apto, y es lo que permitirá pasar, eventualmente, de una etapa de imitación tecnológica a una de exploración y explotación más eficiente, generadora de innovación. Es decir, a un sistema de innovación, propiamente. De aquí la importancia de un enfoque sistémico en política, que permita orientarla a romper el estado disfuncional que prevalece actualmente en este tipo de países (path dependence) ${ }^{6}$ dentro de un enfoque global de política de aprendizaje para la innovación.

\section{Aprender a I nnovar como Objetivo de Política de Vinculación}

En una situación de desvinculación hay que recordar algunas características de la actividad investigadora de importancia para la planeación (Nath y Mrinalini, 2000), relacionadas con el aprendizaje para la innovación:

- Sus productos, resultados y beneficios podrán ser tanto tangibles como intangibles.

- Los vínculos entre investigación y su utilización e impactos no son necesariamente directos ni inmediatos.

- Los centros públicos de I+D son sólo un tipo de actor. Su papel es producir y transferir conocimiento socialmente relevante.

Tomando esto en consideración, la pregunta es cómo comenzar a construir los canales para que fluya el conocimiento que se necesita, en un entorno en el que los distintos grupos de interés han estado secularmente desvinculados y muchos de ellos ni siquiera tienen claro su papel.

\footnotetext{
${ }^{6}$ Path dependence: donde estamos hoy, es resultado lo que ha sucedido en el pasado.
} 
REDES- Revista hispana para el análisis de redes sociales

Vol.17,\#3, Diciembre 2009

http: // revista-redes. rediris.es

A partir de la necesidad de acoplar las distintas dimensiones de la actividad investigadora (científica, tecnológica, educacional, cultural, etc.) con las cambiantes necesidades de conocimiento de la sociedad, un CIU ha de identificar los objetivos estratégicos que le permitan vincularse, así como ciertas buenas prácticas, rutinas y actividades funcionalmente relacionadas que al favorecer directamente el cumplimiento de tales objetivos, incrementen su potencial o aptitud de transferencia de conocimiento, e indirectamente, la construcción de la capacidad de absorción.

Aprender a innovar significa pues, entre muchas otras cosas, que los grupos de interés (o actores) han de aprender a interactuar adecuadamente - a vincularse -, para que fluya el conocimiento del que se nutre la innovación. El diseño de políticas de vinculación dentro de un enfoque sistémico implica identificar y ocuparse de los siguientes elementos: a) su papel dentro del sistema; b) las necesidades de su mercado; c) los saberes y competencias que requiere dominar para responder efectivamente a esas necesidades, de acuerdo con su misión; d) los recursos con los que cuenta; e) tener en cuenta su contexto.

a. Su papel dentro del sistema. El papel de los CIUs es producir y transferir conocimiento. Para que ello ocurra, éste ha de tener calidad social (pertinencia). Es decir, ha de ser científicamente válido y socialmente útil.

b. Orientarse hacia las necesidades de su mercado implica no sólo identificarlas cuidadosamente, sino internalizarlas dentro de su propia estructura organizacional. Esto se reflejará en las diversas dimensiones de la actividad investigadora (investigación científica, desarrollo tecnológico, educación y servicios). Lo que contribuirá a darle credibilidad a la organización, a generar lazos perdurables y un sano clima de confianza.

c. Las competencias adecuadas. Las nuevas tecnologías tienden a ser cada vez más complejas. El conocimiento que produce un $\mathrm{CIU}$ ha de ser lo suficientemente avanzado y relevante como para poder responder oportunamente a las necesidades cambiantes de la sociedad a la que pertenece. También se hace necesario cultivar atributos y competencias adecuadas, como la habilidad para el trabajo en equipo y la interdisciplina. Ellas contribuirán a facilitar el intercambio de conocimiento, un clima de confianza, un lenguaje común con sus contrapartes, etc.

d. Contar con la infraestructura, el capital humano y financiero adecuados, son elementos de política de vinculación fundamenatles para un CIU. Saber manejarlos, es crucial. 
REDES- Revista hispana para el análisis de redes sociales

Vol.17,\#3, Diciembre 2009

http: // revista-redes. rediris. es

e. Tener en cuenta el contexto dentro de la estrategia es vital, para detectar las limitaciones, amenazas y oportunidades en las que el ego se puede vincular con sus alters.

\section{Los Objetivos Estratégicos}

Cuando no existe una cultura de vinculación, el primer gran objetivo general de un $\mathrm{CIU}$ para transferir el conocimiento es orientar la actividad investigadora hacia las necesidades de la sociedad. Todos los otros objetivos estratégicos de política han de estar subordinados a éste. En este nivel micro, los objetivos estratégicos pueden ser aquellas características que el CIU tendrá que alcanzar mediante el cultivo de ciertas buenas prácticas y actividades. En conjunto, ellas contribuirán a establecer un nivel de aptitud más alto para vincularse. Tales actividades formarán los canales o mecanismos por donde fluirá el conocimiento hacia y desde su mercado. Algunas prácticas y actividades contribuirán directamente al establecimiento de vínculos con sus alters, en tanto que otras tendrán un efecto indirecto, de más largo plazo, pero de gran importancia para el engranaje de unas redes con otras.

Los vínculos resultan, en última instancia, de una diversidad de decisiones. Estas a su vez, son afectadas por una serie de factores, en donde suelen entremezclarse infinidad de intereses públicos y privados. En gran medida, las decisiones son facilitadas o limitadas en lo cotidiano - a nivel micro -, por una serie de prácticas o rutinas y normas institucionales específicas para cada tipo de organización, que derivan, a su vez, de sus políticas de desarrollo y estrategias particulares. Las capacidades y competencias para llevarlas a cabo pueden variar con los sistemas tecnológicos y con el contexto específico. Cuando tales prácticas o actividades no están funcionalmente orientadas a objetivos, y no existen las competencias adecuadas, el potencial de interacción de un actor ego disminuye. Cuando los actores ego no interactúan, la aptitud del sistema para innovar, decrece.

En un nivel meso, el engranaje entre las diversas redes ego estará lubricado por el nivel de aptitud para innovar, resultante de las contribuciones de los actores en el nivel micro, como son formación de capital humano adecuado en el caso de los CIUs (para la construcción de la capacidad de absorción), o del establecimiento de instituciones y políticas públicas para el fomento de la innovación y un marco jurídico que asegure el financiamiento sostenido de la I+D en el caso del gobierno, o la integración de cadenas de proveedores en el sector productivo. En el nivel macro, el funcionamiento del sistema de innovación es una expresión del grado de articulación de las múltiples redes que lo integran en los otros niveles (red de redes). De aquí la 
REDES- Revista hispana para el análisis de redes sociales

Vol.17,\#3, Diciembre 2009

http: // revista-redes. rediris.es

importancia de un enfoque sistémico y de la integración del ARS en el diseño de políticas para la innovación.

\section{Redes de Actores y Redes de Actividades}

A través de las interacciones entre los distintos actores, se favorece el uso del conocimiento. Con sus habilidades y motivados por sus intereses históricos, cada uno va lanzando anzuelos, se va enganchando con otros - forman diadas - de donde resultan distintas configuraciones de lo que se conoce como red ego. Del adecuado engranaje de éstas dependerá el buen funcionamiento de la red global (o red de redes) que conforma lo que se conoce como sistema de innovación. A nivel micro, desde el punto de vista de los CIUs, el reto es inducir el cambio del estado actual de vinculación hacia uno más eficiente, de manera sostenida. La conformación de sus redes con otros actores y su configuración es un indicador importante de su aptitud para vincularse. A nivel operativo, el problema es cómo hacerlo. Es decir, cómo construir a nivel de la organización esos canales para intercambiar conocimiento con los diversos grupos de interés, con necesidades diversas y cambiantes e identificar cuáles serán los mecanismos más adecuados en cada momento y situación.

Los canales por donde fluye el conocimiento entre el $\mathrm{CIU}$ y sus usuarios/beneficiarios /clientes forman el entramado "visible" de su red. A través de ella se mueven múltiples intereses públicos o privados. En este ambiente, lo que incrementa verdaderamente la aptitud o potencialidad de vinculación de una organización es el conjunto de prácticas y actividades ligadas a ciertos objetivos estratégicos, a los que es necesario prestar atención.

Los objetivos estratégicos para inducir una dinámica de acercamiento entre el Centro y sus clientes (actuales o potenciales) son "facilitadores" para construir diadas. Tener claros estos objetivos estratégicos es fundamental para incrementar el potencial de transferencia de conocimiento de la organización. Algunos ejemplos de objetivos estratégicos son el cultivo de lazos perdurables; generación de un clima de confianza; construcción de un lenguaje común; interdisciplina participación en redes.

¿Cómo alcanzar esos objetivos estratégicos que favorecen la interacción, es decir la formación de diadas? La institucionalización de buenas prácticas y actividades funcionalmente interconectadas para alcanzar cada uno de los objetivos identificados es el camino. Su interconexión en función de objetivos nos permite pensar el problema en términos de micro redes de actividades funcionalmente relacionadas. Como las actividades y objetivos son para establecer el vínculo, estamos a nivel de diada. Por tratarse de redes intencionales - orientadas a objetivos estratégicos 
REDES- Revista hispana para el análisis de redes sociales

Vol.17,\#3, Diciembre 2009

http: // revista-redes. rediris.es

deseables -, es posible integrar el enfoque teleológico de sistemas complejos para la planeación estratégica, con el ARS (Tabla 1).

Integración de Análisis de Redes Sociales para

Politicas de Innovación: Enfoque Teleológico

\begin{tabular}{|c|c|c|c|c|}
\hline $\begin{array}{c}\text { Nivel } \\
\text { de } \\
\text { análisis }\end{array}$ & Sistema & $\begin{array}{l}\text { Red de } \\
\text { Actores } \\
\text { (Quién } \\
\text { con } \\
\text { quien) }\end{array}$ & $\begin{array}{l}\text { Red de } \\
\text { actividades } \\
\text { Intra diada: } \\
\text { (Cómo) }\end{array}$ & $\begin{array}{c}\text { Objetivos /Atributos } \\
\text { (Para qué) }\end{array}$ \\
\hline MACRO & \begin{tabular}{|l} 
Sistema de \\
Aprendizaje/ \\
Innovación
\end{tabular} & $\begin{array}{l}\text { Red de redes } \\
0 \\
\text { macro red }\end{array}$ & Articulación & $\begin{array}{l}\text { - Nivel de aptitud } \\
\text { adecuado para competi } \\
\text { ó Captura Tecnológica } \\
\text { (catching up) } \\
\text { - Desarrollo basado en } \\
\text { conocimiento }\end{array}$ \\
\hline MESO & Instituciones & $\begin{array}{l}\text { Red } \\
\text { Institucional }\end{array}$ & Engranaje & $\begin{array}{l}\text { - Capacidad de absorción } \\
\text { - Uso eficiente de } \\
\text { conocimiento }\end{array}$ \\
\hline MICRO & Organizaciones & Red ego & Anzuelo & $\begin{array}{l}\text { - Clima de confianza } \\
\text { - Lenguaje común } \\
\text { - Interdisciplina }\end{array}$ \\
\hline
\end{tabular}

Tabla 1.

\section{Micro Redes de Actividades I ntradiada}

Como se ha enfatizado a lo largo de este trabajo, un elemento importante para la articulación de un sistema de innovación (sea nacional, regional, local, sectorial, etc.) es la conformación de redes entre actores, para favorecer el flujo efectivo de conocimiento tecnológico. Por la naturaleza misma del proceso innovador, tales redes han de ser perdurables, pero lo suficientemente flexibles como para permitir el acoplamiento a los requerimientos del cambio tecnológico continuo (catching up).

Dentro de esta dinámica, en el contexto de un país con poca cultura innovadora y una secular desvinculación entre los actores, el objetivo global deseable de la participación en redes en un primer momento sería inducir el rompimiento de la inercia imitadora (path dependence), para favorecer con el tiempo, una actitud innovadora. La tarea sería construir esa capacidad de absorción tecnológica del sector productivo nacional que tanto se requiere para contender con las crecientes presiones mundiales de la competitividad basada en conocimiento. Aquí se trata pues, como se señaló antes, de redes de aprendizaje para la innovación.

Para un centro de I+D universitario - en tanto actor clave como productor y transmisor de conocimiento -, que quiera responder a esa necesidad fundamental del país, una tarea importante es vincularse con su mercado. Para ello tiene que acercarse a él, interactuar continuamente para generar un clima de confianza y credibilidad, que permita detectar a fondo sus necesidades y aportar conocimiento 
REDES- Revista hispana para el análisis de redes sociales

Vol.17,\#3, Diciembre 2009

http: // revista-redes. rediris.es

orientado a ellas (calidad social); para enseñar a detectar las oportunidades que ofrecen las nuevas tecnologías potencialmente disponibles en el mercado; para aprender a traducir problemas industriales en problemas de I+D; para establecer un lenguaje común; para establecer sinergias en los intercambios de conocimiento mediante el trabajo interdisciplinario y en equipo, etc.

Hablar de actividades es hablar de dinámica y posibilidades de cambio. Para alcanzar objetivos como los señalados arriba, un centro de I+D ha de realizar diversas actividades dentro de un proceso estratégico de cambio. Por lo tanto, podemos pensar que en este tipo de redes, para aumentar la probabilidad de que ocurra el vínculo es necesario tomar en cuenta no sólo con quién se relaciona un actor, sino cómo incrementa su potencial y qué actividades realiza para establecer cada una de sus interacciones. Una manera de estudiarlo, planteamos aquí, es hacer un "acercamiento" al interior de la diada, y ver esa peculiar micro red de actividades que le da vida. Es precisamente en este nivel, intradiada, asociado a los problemas, actividades y objetivos de un actor, donde puede intervenir el planificador y el tomador de decisiones de una organización, para inducir el cambio, mediante la implantación de buenas prácticas y actividades estratégicamente orientadas. Las buenas prácticas y actividades institucionalizadas que se implementan a nivel operativo han de pensarse en función de los objetivos estratégicos. Cuando las prácticas o actividades están funcionalmente interconectadas se pueden visualizar como micro redes intradiada (Figura 2).

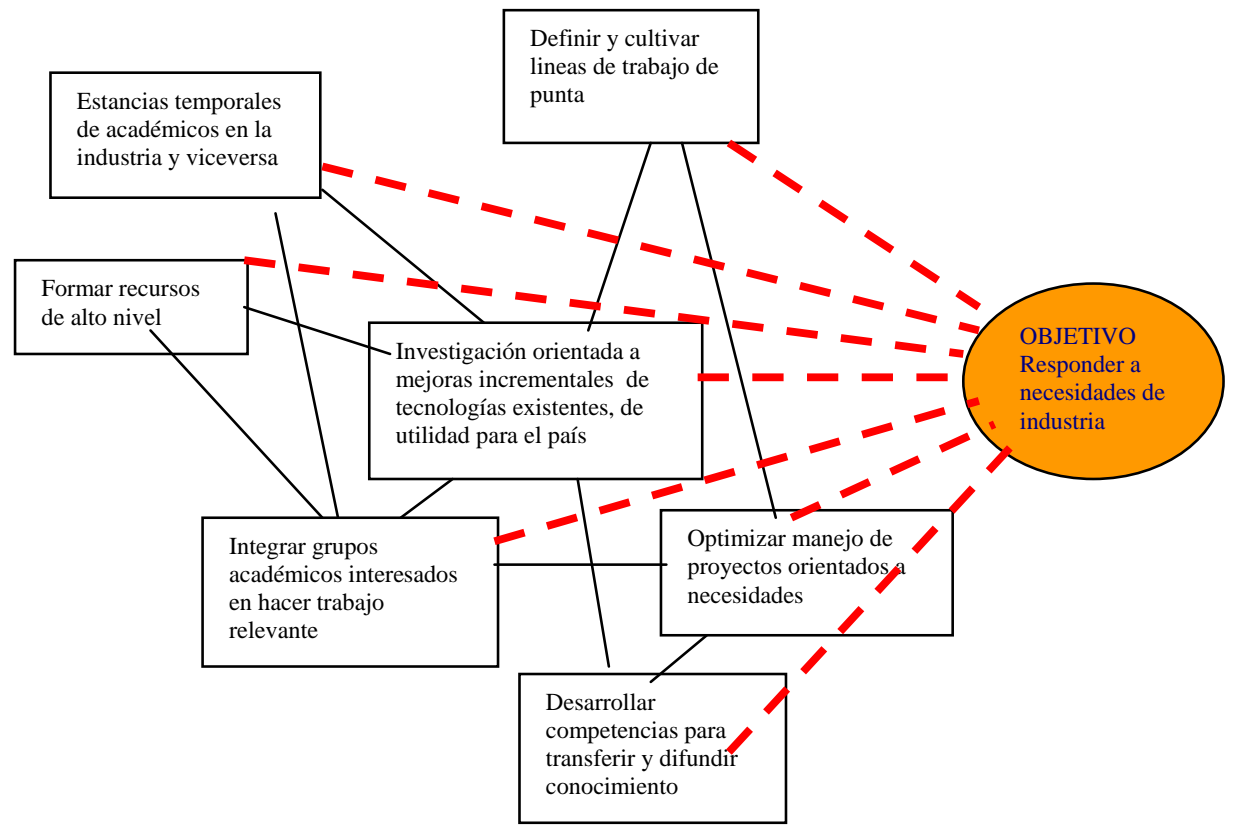

Figura 2. Micro red de actividades intradiada. 
REDES- Revista hispana para el análisis de redes sociales

Vol.17,\#3, Diciembre 2009

http: // revista-redes. rediris.es

En suma, el concepto de micro red de actividades intradiada define el conjunto de prácticas y actividades funcionalmente interconectadas, orientadas a los objetivos estratégicos requeridos para incrementar el potencial de cambio; en este caso, para establecer vínculos entre dos actores.

\section{Sustento del Diseño de la Herramienta}

Una vez propuestos los elementos conceptuales anteriores, el reto es cómo integrarlos en el diseño e implementación de una estrategia que apoye el proceso de cambio de un $\mathrm{CIU}$ hacia la efectividad en relación con la producción, transferencia y difusión de conocimiento relevante. ¿Cómo plantear una dinámica de transferencia de conocimiento con los actores alter? ¿Cómo detectar sus necesidades? ¿Cómo detectar las expectativas? Para ello se ha diseñado una herramienta de diagnóstico (fuerzas y debilidades) que facilite la intervención para el cambio. Dicha herramienta tiene como punto de partida la integración conceptual, empírica y prospectiva de los elementos discutidos anteriormente, y pretende expresar los resultados de manera sencilla. Ofrece una visualización de la línea basal, las tendencias de desempeño y expectativas al respecto, a través de las rutinas que se realizan en la institución, teniendo como punto de referencia las mejores prácticas a nivel internacional. Todas ellas han de ser enmarcadas en los procesos institucionales críticos para la estrategia de vinculación. La herramienta permite, asimismo, identificar aspectos o puntos específicos que tienen posibilidades de intervención, en un nivel de actividades concretas.

En este esquema se identifican y proponen aquellas buenas prácticas reportadas en la literatura mundial (concebidas como buenos arreglos de interacción) que pudieran ser integradas dentro de las políticas institucionales. El Cuestionario, a través de la satisfacción reportada para tales prácticas, permite medir la efectividad de su aplicación. Esto da cuenta de la aptitud institucional para vincularse con el sector productivo.

Tomando en cuenta lo anterior, la herramienta, que hemos llamado "Instrumento de Autoevaluación Institucional" comprende los siguientes elementos:

1. Base de Datos de Buenas Prácticas Internacionales, seleccionadas para el contexto local, para los siguientes procesos organizacionales relacionados con la vinculación: Manejo de la Investigación, Manejo de Capital Humano, Manejo de Servicios y Resultados.

2. Talleres de Aprendizaje y de Consenso.

3. Entrevistas y Cuestionario para recopilación de datos. 
REDES- Revista hispana para el análisis de redes sociales

Vol.17,\#3, Diciembre 2009

http: // revista-redes. rediris.es

En otros trabajos anteriores se ha presentado el sustento teórico general y la metodología para la selección de las Buenas Prácticas (Almanza y Hernández, 2007). Aquí se presenta el sustento para la recopilación de datos del cuestionario con el fin de ilustrar la expresión de la integración conceptual en las preguntas planteadas. La validación empírica de la metodología se efectuó en un centro de investigación universitario en México.

Cabe señalar que el cuestionario contempla preguntas orientadas no sólo a obtener información sobre la estructura, prácticas y actividades actuales de la organización - derivadas de las políticas implantadas anteriormente -, sino a resaltar la percepción que tienen los propios académicos sobre los resultados derivados de ellas, y con quienes desearían interrelacionarse, en un esquema de cambio, (de donde podría configurarse la red deseable). Con esta información se busca hacer palpables las fortalezas y debilidades de la institución derivadas de tales "usos y costumbres", y mostrar a los participantes otras maneras de hacer las cosas, mediante la inclusión en el cuestionario de algunas buenas prácticas reportadas en la literatura - seleccionadas con base en sus posibilidades de aplicación al contexto particular -, que han probado su efectividad para alcanzar los objetivos clave. Se asume que mientras más objetivos se alcancen, mayor será el potencial o aptitud de la organización para vincularse.

El plantearlo así e involucrar en el proceso de evaluación institucional a todo el personal académico que acceda a responderlo, facilita la toma de conciencia no sólo del papel que cada uno juega dentro de la organización, sino del papel de ésta dentro del sistema y las implicaciones que puede tener si se vincula mejor. Adicionalmente, la dinámica participativa - que incluye talleres y charlas previas y posteriores, ofrece la ventaja de que se va preparando el terreno para el cambio, por lo que tendrá más probabilidades de ser aceptado. Desde esta perspectiva, se le puede ver, además, como un valioso instrumento de aprendizaje institucional, consistente con el objetivo de la planeación.

\section{El diseño del instrumento: elementos}

Las actividades relacionadas con la investigación, desarrollo e innovación (I+D) por definición, requieren de muy diversas interacciones entre los involucrados para hacer un uso más eficiente del conocimiento. A medida que se vuelve más compleja y dinámica la producción de tecnología y el proceso de innovación, la participación en redes de los grupos de interés se va volviendo cada vez más la forma natural de interactuar en el mundo. En México, sin embargo, esta tendencia se ha visto frenada en gran medida por el alto grado de desarticulación entre los 
REDES- Revista hispana para el análisis de redes sociales

Vol.17,\#3, Diciembre 2009

http: // revista-redes. rediris.es

diversos actores. Por lo que toca a las instituciones de educación superior, la vinculación ciencia - industria sigue siendo el gran reto. Aunque es claro que se percibe la necesidad impostergable de vincularse con el sector productivo, no se tiene muy claro cómo romper las inercias y cómo inducir el cambio en sus centros de investigación, para incrementar su aptitud de vinculación.

Tomando en cuenta que se desea transferir de manera más eficiente el conocimiento que ahí se produce, y que ello requiere del cultivo de ciertas características de la actividad investigadora, se hace necesario considerar lo siguiente en el diseño del instrumento:

- Con quienes se relaciona el Centro actualmente (red ego real), para identificar sus fuerzas y debilidades.

- Con quiénes desea relacionarse a futuro (red ego deseable), para el logro de su misión y visión de desarrollo.

- Cómo establecer sus diadas de manera efectiva: Identificar los objetivos y características estratégicas

- Identificar los canales y mecanismos más adecuados a su situación y contexto, para cada tipo de relación con sus clientes, usuarios, patrocinadores o beneficiarios (alters), para que fluya eficientemente el conocimiento (actividades y prácticas).

De manera breve, el desarrollo de este instrumento de planeación ofrece un marco estratégico para 1 ) el diagnóstico o línea basal en términos de las actividades y prácticas que realiza; 2) ofrecer el posicionamiento de la organización en torno a su aptitud para innovar, asociada a la calidad y cantidad de interconexiones que presenta; 3) identificar expectativas o posición deseable a través del nivel de aceptación de las buenas prácticas y 4) orientar la definición de una estrategia institucional al identificar huecos en las prácticas funcionalmente interconectadas que serían necesarias para cumplir los objetivos que la organización se haya propuesto.

\section{Método de Recolección de Datos}

El corazón del instrumento de auto evaluación institucional es, esencialmente, un cuestionario para ser aplicado al personal académico del centro en cuestión, complementado con información proveniente de los talleres y de la documentación oficial $^{7}$. El instrumento está teóricamente sustentado dentro del paraguas

7 La mayoría de las preguntas buscan capturar las percepciones de los académicos integrantes del centro en estudio, para asegurar que el cuestionario sea completado fácilmente. Todas las respuestas son referidas de manera grupal o en forma anónima en el reporte final. 
REDES- Revista hispana para el análisis de redes sociales

Vol.17,\#3, Diciembre 2009

http: // revista-redes. rediris.es

conceptual de Sistemas Complejos de Innovación, adaptado al problema de la articulación ciencia - industria en países poco innovadores, centrado como se señaló en la primera parte, dentro de una perspectiva de aprendizaje para la innovación como sistema de transición. De aquí es importante recordar que la vinculación es definida en sentido amplio (Almanza y Hernández, 2007) .

Para el diseño del cuestionario y el tipo de información requerida, se tomaron en cuenta los objetivos globales del nuestra línea de investigación. De entre ellos, destacamos aquí únicamente el relacionado con la integración del ARS al instrumento para facilitar y/o enriquecer la interpretación de los resultados. En este sentido, se consideró la necesidad de obtener información para:

1. Tener una apreciación global de la situación actual y deseable del Centro en estudio. Es decir, caracterizar los grupos de interés para el Centro, que estén actualmente vinculados con él (red ego actual), y con quienes desea establecer vínculos para configurar una red de relaciones más adecuada para el cumplimiento de su misión y su visión estratégica (red ego deseable).

2. Para un nivel de análisis más profundo - a nivel de diada -, es importante conocer la forma del manejo de la vinculación del centro. Es decir, cómo se vincula actualmente, qué prácticas, rutinas o actividades realiza o deja de realizar, para dar vida o para frenar el establecimiento de cada uno de esos vínculos, y ser contrastados con la teoría y la experiencia de otras instituciones en el mundo. Es decir, con aquellas buenas prácticas, rutinas o actividades institucionales reportadas en la literatura que pudieran ser estratégicamente implantadas para desarrollar determinadas características (targets) reconocidas como clave para incrementar la aptitud de vinculación de los CIUs.

\section{Tres Niveles de Análisis}

Para el diseño del cuestionario, desde nuestra perspectiva e interés por la aplicación del ARS a la planeación de políticas de fomento a la innovación, conviene distinguir tres niveles de análisis (Tabla 1). Un primer nivel amplio y por demás complejo, es el sistema o red de innovación (nacional, regional, local, sectorial, etc.), que puede verse como una gran "red de redes ego" ${ }^{\prime}$. En este nivel, además de otros factores, el buen funcionamiento depende en gran medida del grado de articulación o engranaje adecuado entre las redes que establecen los diversos actores entre sí, y el entendimiento y cumplimiento de los papeles o funciones que

8 La vinculación en sentido amplio permite dar cabida a la amplia gama de "productos", resultados y consecuencias derivadas del manejo y actividad de la investigación

9 Una red completa contiene una red egocéntrica por cada objeto dentro de ella (Marsden, 2002; Marsden, 2005). 
REDES- Revista hispana para el análisis de redes sociales

Vol.17,\#3, Diciembre 2009

http: // revista-redes. rediris.es

a cada uno de ellos le corresponde. Es esta red completa la que debe ser tejida en países poco innovadores, para la articulación de los sistemas de innovación.

En un segundo nivel, el énfasis recae en las interacciones que establece una organización o actor del sistema de innovación. Este tipo de red -red ego- es importante para tener una apreciación de la situación de un actor particular, y habla de su comportamiento preciso dentro del sistema, al destacar con quiénes se conecta, teniendo presente que las acciones intencionales estructuralmente limitadas de los individuos pueden afectar la estructura social en la que está inmerso (Sanz Menéndez, 2003).

En un tercer nivel de análisis, que a menudo se ignora, el énfasis pasa a tratar de entender cómo se conecta la organización con quién. Es decir, qué actividades se han de realizar para dar vida al vínculo. Ello requiere tejer más fino, hacer un acercamiento más preciso, microscópico, al interior de la diada y amplificar eso que hemos llamado micro redes de actividades, a través de las cuales se desarrollarán aquellas características requeridas por los actores para que ocurra la anhelada vinculación. Al respecto, es importante destacar que no nos estamos enfocando únicamente a pares de relaciones diádicas, sino al proceso de aprendizaje de un $\mathrm{CIU}$ con sus alters para llegar a construir una red completa de interacciones en materia de innovación.

En este proyecto, respondiendo al interés particular de un Centro de I+D por implementar el cambio para incrementar su aptitud de vinculación con el sector productivo, y nuestro interés en desarrollar el concepto de micro-redes de actividades intradiada -, nos centramos en el segundo y sobre todo, en el tercer nivel de análisis, y es en función de esta necesidad, que se orienta el cuestionario. Por la extensión de este, lo que se presenta aquí son únicamente algunos elementos de interés para ilustrar la secuencia lógica y el potencial de aplicación del ARS que se vislumbra, para problemas complejos y dinámicos de políticas, de estrategia y de innovación, por mencionar algunos.

En la Tabla 2 se ilustra la relación entre dos procesos organizacionales de importancia para la vinculación - el Manejo de la Investigación y el Manejo del Capital Humano - con sus buenas prácticas y actividades funcionalmente asociadas con los objetivos estratégicos de la organización, de acuerdo con la metodología del Benchmarking. Las buenas prácticas y actividades son plasmadas en el cuestionario. De esta manera, las respuestas darán la configuración actual y deseable de las micro-redes de actividades intradiada y de la red ego del CIU. Con base en ellas, podrá sacarse un diagnóstico de las fortalezas y debilidades de la 
REDES- Revista hispana para el análisis de redes sociales

Vol.17,\#3, Diciembre 2009

http: // revista-redes. rediris.es

organización, y sustentar las medidas de corrección requeridas, con la ayuda de la Base de Datos sobre Buenas Prácticas Internacionales para construir la red deseada.

NIVEL MACRO

Objetivo Global de largo plazo (indirecto)

Contribuir a la articulación del Sistema Nacional de Aprendizaje para la Innovación

NIVEL MESO

Objetivo global de mediano plazo (indirecto)

Contribuir a incrementar la capacidad de absorción tecnológica nacional

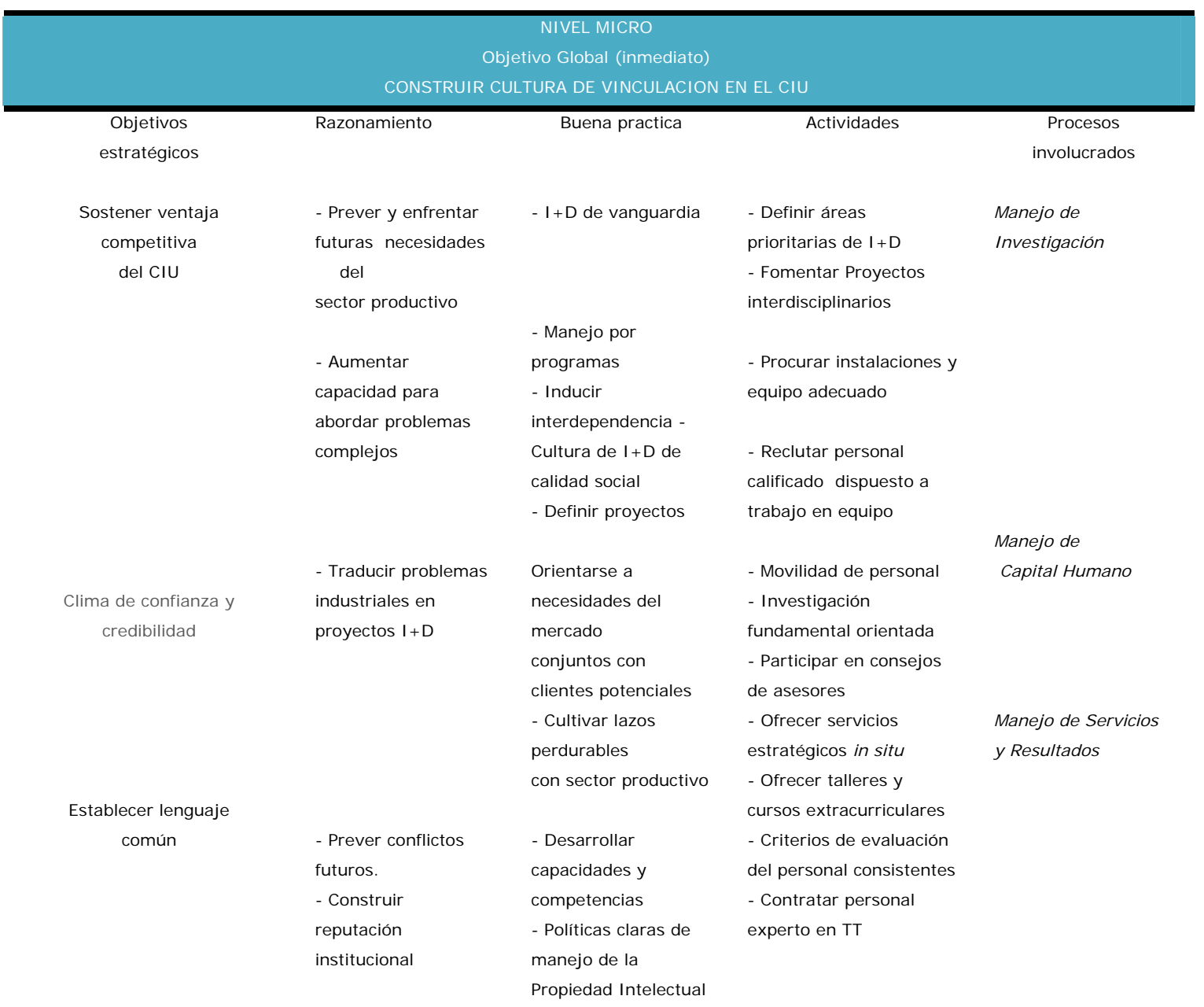

Tabla 2. Conceptualización del Cuestionario: articulación de Objetivos, Buenas Prácticas, Actividades y Procesos 
REDES- Revista hispana para el análisis de redes sociales

Vol.17,\#3, Diciembre 2009

http:// revista-redes. rediris.es

\section{Ejemplos de preguntas}

Es importante cuidar que el cuestionario sea consistente con los objetivos estratégicos de la planeación. La información que se espera obtener permitirá conocer las prácticas y actividades habituales y deseables de la institución y a través de ellas, identificar las fortalezas y debilidades que se plasmarán en el diagnóstico. En lo que sigue se muestran unos cuantos ejemplos de preguntas, con carácter meramente ilustrativo.

Para el objetivo estratégico "Orientarse a las Necesidades del Sector Productivo":

PREGUNTA: La identificación de necesidades de grupos de destinatarios (sectores, cámaras, gremios) es principalmente decidida por:

1. La determinación de necesidades la hacen los académicos, por iniciativa personal

2. Se hace por el Laboratorio considerando primordialmente las capacidades del mismo (technology push)

3. Se consideran principalmente las necesidades del cliente (market pull)

4. Se hace monitoreo e investigación de mercado, por la Dirección apoyada por aportaciones de los Laboratorios

5. Resulta de la participación de los académicos en consejos industriales

6. Considera la opinión de su consejo de asesores industriales

7. Es mediada por el Coordinador de Vinculación

\section{1, 2 = Malas prácticas $\quad 3-7=$ Buenas Prácticas}

\section{PREGUNTA: Las actividades para identificar necesidades de clientes} individuales incluyen:

1. Se da consultaría gratuita in situ

2. Se organizan clínicas tecnológicas y seminarios

3. Se ofrecen programas de suscripción mediante los cuales el servicio a los suscriptores es subsidiado

4. Se organizan estancias de estudiantes en la industria

5. Se organizan estancias de personal de la industria en el Centro

6. Es mediada por el Coordinador de Vinculación

$1-6=$ Buenas actividades

Para credibilidad, generación de confianza, capacidad oportuna de respuesta, calidad social de la investigación, etc:

PREGUNTA: Los atributos que mejor caracterizan a su laboratorio 
REDES- Revista hispana para el análisis de redes sociales

Vol.17,\#3, Diciembre 2009

http://revista-redes. rediris.es

1. Vanguardia tecnológica

2. Pertinencia

3. Confiabilidad en los resultados

4. Cumplimiento en los tiempos de entrega

5. Trascendencia de los resultados

6. Calidad Flexibilidad capacidad para asumir nuevos retos

7. Responsabilidad

8. Prestigio

\section{1 - 8 Buenas Prácticas}

\section{Discusión y Conclusiones}

En este trabajo se ha presentado el sustento del diseño de un instrumento de planeación estratégica teóricamente sustentado, que facilita la intervención hacia el cambio. Enmarcado en el enfoque de sistemas complejos y el ARS, el instrumento tiene como objetivo inicial determinar el nivel de aptitud de un CIU para vincularse, y como objetivo final, inducir el aprendizaje para la innovación.

Es un hecho que una organización en red propicia el proceso de innovación tecnológica y que a su vez, ésta da forma a los cambios subsecuentes en la red. Este proceso, de carácter coevolutivo, es en sí un proceso de aprendizaje. Inducir este proceso de aprendizaje colectivo a través de la interacción es sin duda, una tarea fundamental para el desarrollo basado en conocimiento.

En países poco innovadores, como México, el uso eficiente del conocimiento es limitado. De aquí nuestra propuesta para enfocar el esfuerzo de intervención política basada en el concepto de sistema de aprendizaje para la innovación, como sistema de transición hacia la articulación progresiva de un verdadero Sistema de Innovación. Se ha partido de la idea de que para que fluya el conocimiento que nutre a la innovación, cada actor - desde sus distintas funciones- ha de aprender a interactuar adecuadamente para hacer su aportación y favorecer así, una dinámica de aprendizaje y la articulación de redes de conocimiento. Las actividades y objetivos para el proceso de articulación pueden tener diferentes expresiones en los distintos niveles sistémicos (micro, meso, macro), lo importante es que ellas sean consistentes y articulables con el objetivo global perseguido. Así, la vinculación a nivel micro estará relacionada con la construcción de la capacidad de absorción a nivel meso y ésta, a su vez, con la articulación de red de redes o sistemas de conocimiento, etc. Consecuentemente, la identificación y análisis de los elementos que dan vida a la interacción, desde esta perspectiva, se vuelve fundamental para la intervención política. Abrir el Análisis de Redes Sociales (ARS) 
REDES- Revista hispana para el análisis de redes sociales

Vol.17,\#3, Diciembre 2009

http: // revista-redes. rediris.es

hacia el interior de la diada es un campo muy atractivo no sólo para entender esta dinámica, sino para utilizarlo como herramienta de intervención política. Una manera de hacerlo, es la presentada en este trabajo, con la propuesta del concepto de micro redes de actividades intradiada.

La propuesta de abordar problemas complejos y dinámicos de política y estrategia de innovación integrando el ARS, parte de la necesidad -por demás urgente-, de instrumentar acciones que articulen de manera coherente y progresiva a los actores para conformar un verdadero Sistema Nacional de Innovación. Asimismo, surge del convencimiento de que el ARS constituye tanto un enfoque como una herramienta adecuados para analizar las interacciones actuales y procesos que emergen del Sistema de Aprendizaje Para la Innovación, aquí propuesto. Como enfoque, nos permite abordar el proceso de tejer la red desde el nivel de diada, redes ego y redes completas; igualmente, permite incorporar en el análisis los componentes básicos de la red: los actores y sus características, así como la parte dinámica de los mecanismos, motivaciones y modificaciones que les dan vida en el tiempo, a través de su evolución; y también, de manera muy importante, colectar la información de manera sistemática.

Como herramienta, su aplicación en todos los niveles (diada, red ego, redes completas) permite incorporar al análisis propiedades no evidentes de la estructura de la red y por supuesto, su representación gráfica. Para el caso que nos ocupa, la utilización del ARS como herramienta apoya la orientación de la intervención hacia el aprendizaje - que es la propiedad emergente más importante del Sistema de Innovación-. De esta manera, la inclusión del concepto de micro redes de actividades intradiada aquí propuesto abre un gran potencial de aplicación del ARS, ya que al integrarle el aspecto teleológico permite el paso de la metáfora al de la intervención en el diseño de políticas.

\section{Bibliografía}

Almanza, S. y Hernandez, H. (2009). "Sustento Teórico - Metodológico para favorecer las Relaciones Ciencia - Industria desde la Universidad", en Globalización, Conocimiento, Desarrollo, en A. Dabat, M.A. Rivera, y Rodríguez, J. (Eds), Porrúa- UNAM, México.

Almanza, S., González, R.L. and Hernández, H. (2004). "Linking academic research centers with the productive sector in developing countries: a strategic planning tool to improve absorptive capacity", International Workshop, Public research institutions, international business, and technological and economic catch up in 
REDES- Revista hispana para el análisis de redes sociales

Vol.17,\#3, Diciembre 2009

http: // revista-redes. rediris.es

developing regions, Faculty of Political Science, University of Catania, 19-20, march, 2004.

Arnold, E. \& Balázs. K. (1998) Methods in the Evaluation of Publicly Funded Basic Research. A review for OECD. Technopolis, U.K. http://www.technopolis.co.uk/reports

Arocena, R. and Sutz, J. (2002). "Innovation systems and developing countries", DRUID, Working Paper № 02-05, Denmark.

(http://www.druid.dk/uploads/tx_picturedb/wp02-05.pdf).

Cohen,W.M and Levinthal, D. (1990). “Absorptive capacity: a new perspective on learning and innovation" in Administrative Science Quarterly, Vol. 35, pp. 128-52.

Conway, S., Jones, O. and Steward, F. (2001) "Realising the potential of the social network perspective in innovation studies", in O. Jones and S. Conway (eds) Social interaction and organizational change: Aston perspectives on innovation networks, Imperial College Press: London, 349-366.

Daghfous, A. (2004). "Absorptive capacity and the implementation of knowledgeintensive best practices", SAM Advanced Management Journal, Spring 2004, 69, 2, ABI/INFORM Global.

Dahlman, C. and Nelson, R. (1995). "Social absorption capability, national innovation systems and economic development", in D.H Perkins, and B.H. Koo (eds.), Social capability and long-term growth, MacMillan Press: England.

David, P.A, and Foray, D. (1995). "Accesing and expanding the science and technology base", STI Review, 16.

Dodgson, M. (2000). The Management of Technological Innovation: A International and Strategic Approach, Oxford University Press: Oxford.

Kline, S.J. and Rosenberg, N. (1986). "An overview of innovation" in R. Landau and N. Rosenberg (eds) The positive sum strategy. Harnessing technology for economic growth, National Academy Press: Washington, D.C. pp. 275-305.

Lundvall, B-A. (1985). "Product innovation and user-producer interaction", Industrial Development Research Series $N^{\circ} 31$, Aalborg University Press: Aalborg.

Lundvall, B-A. (1988). “Innovation as an interactive process: from user-producer interaction to the national system of innovation", in G. Dosi, C. Freeman, R. Nelson, G. Silverberg and L. Soete (eds), Technical Change and Economic Theory, Pinter Publishers: London. 
REDES- Revista hispana para el análisis de redes sociales

Vol.17,\#3, Diciembre 2009

http: // revista-redes. rediris.es

Lundvall, B-A. (1996). "The social dimensions of the learning economy", DRUID Working Paper No. 96-1, Denmark.

Lundvall, B-A. (1996a). "The university in the learning economy", DRUID Working Paper, No 02-06, Denmark. (http://www.druid.dk/uploads/tx_picturedb/wp0206.pdf).

Lundvall, B-A. and Borras, S. (1999). The Globalising Learning Economy: Implications for Innovation Policy, Brussels, DG XII.

Lundvall, B-A. (2004). "The economics of knowledge and learning" in J.L. Christensen and B-A Lundvall (eds.) Product Innovation, Interactive Learning and Economic Performance, Elsevier:Amsterdam, (pp. 21-42).

Mansfield, E. and Lee, J.Y. (1996). "The modern university: contributor to industrial innovation and recipient of industrial R\&D support", Research Policy, 25, pp. 10471058.

Mansfield, E. (1997). “Links Between Academic Research and Industrial Innovations", in P. David, and E. Steinmueller (eds.), A Production Tension: University-Industry Collaboration in the Era of Knowledge-Based Economic Development, Stanford University Press: Palo Alto.

Marsden, P. (2002). "Egocentric and socioeconomic measures of network centrality", Social Networks, 24: 407-422.

Marsden, P. (2005). "Recent developments in network measurement", in P.J. Carrington et al (eds) Models and methods in social network analysis, Cambridge University Press: New York, pp.8-30.

Narula, R. (2004). “Understanding absorptive capacities in an Innovation System context: consequences for economic and employment growth" MERIT Infonomics Research Memorandum Series. (http://www.merit.unimaas.nl; http://www. infonomics.nl).

Nath, P. and Mrinalini, N. (2000). "Benchmarking the best practices of noncorporate R\&D organizations", Benchmarking: An International Journal, 7, 2, pp. 86-97.

Porter, M. E., Schwab, K. and Sala-I-Martin, X. (eds) (2007). The Global Competitiveness Report 2007-2008, MacMillan: England.

http://www.gcr.weforum.org.

Prahalad, C.K and Hamel, G. (1990). "The Core Competence of the Corporation", Harvard Business Review, May/June, 68, 79-91. 
REDES- Revista hispana para el análisis de redes sociales

Vol.17,\#3, Diciembre 2009

http: // revista-redes. rediris.es

Rycroft, R.W and Kash, D.E. (1999). The Complexity Challenge: Technological Innovation for the 21st Century, Pinter: London.

Sanz-Menéndez, L. (2003). “Análisis de redes sociales: o como representar las estructuras sociales subyacentes", Apuntes de Ciencia y Tecnología, $N^{\circ} 7$, junio de 2003, España, pp. 21-29.

Sundbo, J. (1998). The Theory of Innovation: entrepreneurs, technology and strategy, Edward Elgar: Cheltenham, UK.

Van der Meulen, B. and Rip, A. (2000). "Evaluation of societal quality of public sector research in the Netherlands", Research Evaluation, Vol.9, Number 1, pp. 1125. 